\title{
CHANGES IN EXTRACELLULAR SPACE OF THE MOUSE CEREBRAL CORTEX DURING HYDROXYADIPALDEHYDE FIXATION AND OSMIUM TETROXIDE POST-FIXATION
}

\author{
A. VAN HARREVELD AND F. I. KHATTAB \\ Kerckhoff Laboratories of the Biological Sciences, California Institute of Technology, \\ Pasadena, California, U.S.A.
}

\begin{abstract}
SUMMARY
Perfusion of the cerebral cortex of mice with a 4.5 and $12.5 \%$ hydroxyadipaldehyde (HAA) solution in a cacodylate buffer caused a biphasic change in the tissue conductivity. After a latency of a fraction of a minute the cortical conductivity dropped markedly, reaching a minimum in $\mathrm{I}^{\cdot} 5^{-2} \mathrm{~min}$. Then the conductivity increased again. Electron micrographs (EMs) of material perfused with HAA for 1 5-20 min and post-fixed with osmium tetroxide showed electrontransparent swollen structures, some of which could be identified as dendritic. The extracellular space consisted of $100-200 \mathrm{~A}$ slits between the tissue elements and larger spaces in bundles of small profiles (unmyelinated axons). Cortex frozen after 2 min perfusion with HAA and subjected to substitution in acetone containing $2 \% \mathrm{OsO}_{4}$ at $-85{ }^{\circ} \mathrm{C}$ showed swollen (dendritic) structures and a paucity of extracellular material in accordance with the conductivity drop. Often tight junctions between the tissue elements were present. Tissue frozen after $15-20 \mathrm{~min}$ of HAA perfusion when the conductivity had increased again yielded EMs which were characterized by an abundance of extracellular space between the small profiles. The mitochondria in the swollen (dendritic) structures were enormously enlarged. Cortex perfused for $15-20 \mathrm{~min}$ with $\mathrm{HAA}$, post-fixed with $\mathrm{OsO}_{4}$ and then freeze substituted produced EMs resembling those of tissue fixed in the same way but not subjected to freeze substitution. The examination of the fixation process by freeze substitution demonstrated a sequence of major changes in the fluid distribution of the tissue which precludes any direct relationship between the spaces in the normal and fixed tissue.
\end{abstract}

\section{INTRODUCTION}

Torack ( $1965, \mathrm{I} 966)$ observed in brain tissue fixed by perfusion with 4.5 and $12 \cdot 5 \%$ solutions of hydroxyadipaldehyde (HAA) in a cacodylate buffer and post-fixed with osmium tetroxide much larger extracellular spaces than when glutaraldehyde was used for fixation. An inspection of the published electron micrographs (EMs) showed that the extracellular material is not uniformly distributed. At the places where neuronal structures and glia come in contact narrow, $150-200 \AA$ gaps are seen between these elements. The extracellular space is mainly present in bundles of small tissue profiles (probably unmyelinated axons). This distribution is similar to that found in EMs of brain tissue subjected to freeze substitution (Van Harreveld, Crowell \& Malhotra, 1965; Van Harreveld \& Malhotra, 1967). The space distribution observed in EMs prepared with the latter method is believed to be a more faithful representation of the extracellular space in the living tissue than that observed in EMs prepared by conventional fixation methods. The similarity of the extracellular space in HAA fixed and 
freeze substituted material suggested therefore the possibility that fixation of the tissue with HAA is able to preserve the space distribution of the living tissue.

To investigate this possibility impedance measurements of the cerebral cortex were performed during HAA perfusion. A decrease in tissue conductivity (reciprocal of the impedance) as occurs, for instance, during asphyxiation of the cerebral and cerebellar cortices has been considered as an indication of a movement of extracellular electrolytes, accompanied by water to maintain osmotic equilibrium, into the intracellular compartment. This postulate is based on the fact that the intracellular electrolytes are surrounded by membranes of relatively high resistance which prevent intracellular ions from participating freely in the transport of the currents used for the impedance measurements. Such currents are therefore mainly carried by extracellular electrolytes and a loss of these ions can be expected to result in a drop in tissue conductivity (Van Harreveld \& Ochs, I956; Van Harreveld, I96 I, I966). The demonstration of a movement of chloride into certain cellular elements during the conductivity loss suggested that the extracellular electrolytes were taken up by the intracellular compartment (Van Harreveld \& Schadé, 1959; Van Harreveld, 196I). A swelling of the same tissue elements indicated that the electrolyte movement is accompanied by a transport of water into the intracellular compartment (Van Harreveld, 1957, 1961). A conductivity drop accompanied by a fluid and electrolyte shift was observed not only during asphyxiation, but also during spreading depression (Leão \& Ferreira, 1953; Freygang \& Landau, 1955; Van Harreveld \& Ochs, 1957; Ranck, 1964; Van Harreveld, 1958; Van Harreveld \& Schadé, 1959). During glutaraldehyde fixation a drop in the conductivity of the cerebral cortex was demonstrated (Nevis \& Collins, 1967; Van Harreveld \& Khattab, I968).

Additional support for an asphyxial electrolyte and fluid movement was obtained from a study of the EMs of cerebellar and cerebral cortices prepared by freeze substitution. Electron micrographs of these tissues showed an appreciable extracellular space when frozen shortly (within $30 \mathrm{sec}$ ) after circulatory arrest. This space had almost disappeared when the freezing was preceded by 6-8 min asphyxiation (Van Harreveld et al. 1965; Van Harreveld \& Malhotra, 1967). Spreading depression had a similar effect (Van Harreveld \& Khattab, I967). In agreement with the drop in conductivity of the cerebral cortex during glutaraldehyde perfusion it could be shown that this procedure also causes a marked reduction of the extracellular space. During the subsequent post-fixation with osmium tetroxide which (when carried out by perfusion of the tissue) causes an increase in tissue conductivity, the creation of a new extracellular space was demonstrated in the form of the $100-200 \AA$ slits between the tissue elements typical for $\mathrm{OsO}_{4}$ treated material (Van Harreveld \& Khattab, i968).

In the present investigation the conductivity changes of the cerebral cortex during HAA perfusion were examined which could be expected to yield information about electrolyte and fluid shifts during the fixation with this compound. Furthermore, the cortex was subjected to freeze substitution at crucial points during the impedance changes caused by HAA fixation of the tissue. 


\section{METHODS}

Albino mice narcotized with urethane were used in all experiments. The perfusion method was described previously in detail (Van Harreveld \& Khattab, I968). A cannula was tied into the abdominal aorta, pointing cranially. Immediately before the start of the perfusion the thorax was opened and the right heart incised. The preparation was perfused at a pressure of $13 \mathrm{~cm}$ of mercury, first for $10 \mathrm{sec}$ with Ringer's solution then with the fixative. The calvarium and the dura were removed over one hemisphere to prevent a pressure build up in the brain case due to a possible swelling of the tissue during the fixation. Both the exposed and unexposed cerebral cortices were used in experiments in which the tissue was not frozen. For experiments in which the tissue was subjected to freeze substitution only the exposed cortex could be used.

The fixative usually consisted of a $4.5 \%$ solution of HAA in a $0 . \mathrm{I} \mathrm{M}$ sodium cacodylate buffer at $\mathrm{pH} 7.4$ (Torack, $\mathrm{I}^{6} 5$ ) kept at $38^{\circ} \mathrm{C}$. To make the specific resistance of the solution similar to that of the plasma, sodium chloride was added $(6 \mathrm{~g} / \mathrm{l}$.). The specific resistance of the fixative was $69.5 \Omega \mathrm{cm}$ at $38^{\circ} \mathrm{C}$; its osmoconcentration $65^{\circ} \mathrm{m}$ osmol. Perfusion was continued for I $^{-20} \mathrm{~min}$. Often swelling of the brain, as shown by a protrusion of the exposed hemisphere, was observed. In some instances the brain was perfused with a I $2.5 \%$ solution of HAA in the buffer. The EMs were not markedly different from those of material fixed with $4.5 \%$ HAA solution.

After perfusion blocks were cut from the cerebral cortex which were post-fixed by placing them without washing in a $2 \%$ solution of $\mathrm{OsO}_{4}$ in the cacodylate buffer kept at $4{ }^{\circ} \mathrm{C}$ for $2 \mathrm{~h}$. The blocks were then dehydrated in ethanol and embedded in Maraglas (Freeman \& Spurlock, 1962). Thick sections were cut on an LKB Ultrotome which were studied under the light microscope after staining with methylene blue and azure II (Richardson, Jarett \& Finke, I960). Thin sections stained with lead citrate (Reynolds, 1963) were viewed with a Philips EM zoo electron microscope.

In other experiments perfusion with HAA was combined with freeze substitution of the cortex at $-85{ }^{\circ} \mathrm{C}$ in acetone containing $2 \% \mathrm{OsO}_{4}$. The technique of freezing of the tissue on a silver surface at $-207^{\circ} \mathrm{C}$, and the method of substitution were described previously (Van Harreveld \& Crowell, I964; Van Harreveld et al. 1965). Also preparations post-fixed with $\mathrm{OsO}_{4}$ after the HAA perfusion were submitted to freeze substitution. In these experiments acetone without $\mathrm{OsO}_{4}$ was used for the substitution.

The tissue impedance was measured with small silver/silver chloride electrodes placed on the cortical surface. The measurements were carried out with an impedance bridge at $1000 \mathrm{c} / \mathrm{sec}$ (Van Harreveld $\&$ Ochs, 1956). To prevent impedance changes due to temperature fluctuations the measurements were performed in a room kept at $3^{8}{ }^{\circ} \mathrm{C}$ with perfusion fluids warmed to the same temperature. A total of $5^{\circ}$ mice were used in this investigation. 


\section{RESULTS}

\section{Impedance changes during perfusion with hydroxyadipaldehyde}

In Fig. I A the conductivity (reciprocal of the impedance) is plotted (mhos $\times 10^{6}$ ) against time $(\mathrm{min})$ before and during perfusion with HAA $(4.5 \%)$. The conductivity drops after a short latency (fraction of a minute). This latency is shorter than that of the asphyxial conductivity drop ( $\mathrm{I}-2 \mathrm{~min}$ in mice, Van Harreveld \& Malhotra, 1967) indicating that it is the fixative and not $\mathrm{O}_{2}$ deprivation which causes the conductivity change. The conductivity reaches a minimum of $6 \mathrm{r} \%$ of the original value in $\mathrm{r} \cdot 5 \mathrm{~min}$ and then starts to rise slowly to reach after $\mathrm{I} 2 \mathrm{~min}$ a value of $96 \%$ of the preperfusion figure. In most experiments it was possible to measure the cortical impedance for
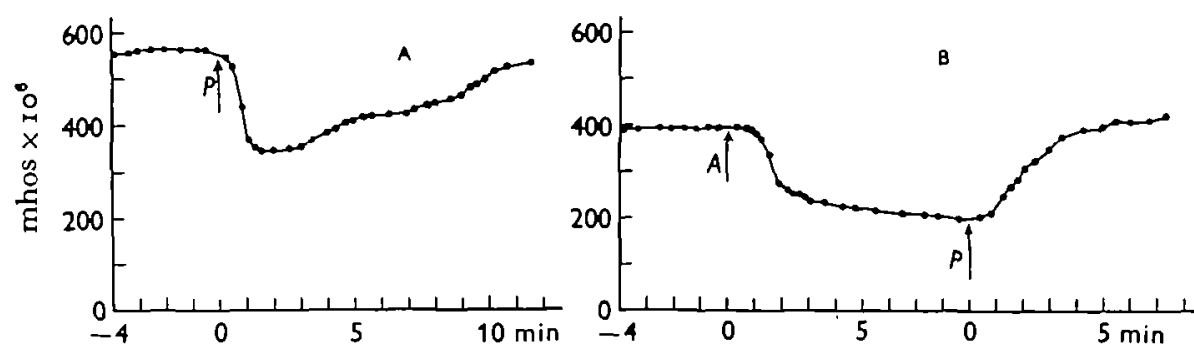

Fig. I. A, conductivity changes of the mouse cerebral cortex during perfusion with hydroxyadipaldehyde $(4.5 \%)$. The perfusion starts at the arrow $P$. B, conductivity changes during asphyxiation of the mouse cortex, followed by perfusion with adipaldehyde. Asphyxiation starts at the arrow $A$; perfusion at the arrow $P$.

7-10 min only since at that time fluid started to accumulate on the cortical surface. This course of the conductivity changes during perfusion with HAA is quite different from that observed during glutaraldehyde perfusion. The latter caused a minimum in 4-5 min that was maintained for the ensuing $15 \mathrm{~min}$ of perfusion. Osmium tetroxide perfusion caused biphasic conductivity changes which were similar to those observed during HAA fixation (Van Harreveld \& Khattab, I968).

Figure I B shows the conductivity changes when the circulation was arrested for 9 min, followed by a $7 \mathrm{~min}$ perfusion with HAA. The asphyxiation caused a marked drop in conductivity, to $49 \%$ of the preasphyxial value. The subsequent perfusion with HAA increased the conductivity again to close to the original figure (106\%) in $7 \mathrm{~min}$. Also in this experiment the conductivity changes during HAA perfusion were different from those during perfusion with glutaraldehyde. The latter did not enhance the conductivity reduced by asphyxiation. The HAA conductivity changes were similar to those produced by $\mathrm{OsO}_{4}$ which also caused an increase in conductivity of asphyxiated tissue (Van Harreveld \& Khattab, I968).

Eighteen similar experiments are summarized in Table $\mathrm{I}$ in which the brain was perfused either without preceding asphyxiation or after 8 min of circulatory arrest. Perfusion both with $4.5 \%$ and $12.5 \%$ HAA solution caused a marked initial drop in conductivity followed by an increase. The conductivity of all asphyxiated preparations increased during perfusion. 
Table I. Conductivity, expressed as a percentage of the value determined in the normal oxygenated cortex, during perfusion with hydroxyadipaldehyde and after asphyxiation followed by perfusion

\begin{tabular}{|c|c|c|c|c|c|c|}
\hline \multirow[b]{2}{*}{$\begin{array}{c}\text { HAA } \\
\text { Concentration }\end{array}$} & \multirow[b]{2}{*}{ No. } & \multicolumn{2}{|c|}{$\begin{array}{l}\text { Perfused immediately } \\
\text { after circulatory arrest }\end{array}$} & \multirow[b]{2}{*}{ No. } & \multicolumn{2}{|c|}{$\begin{array}{c}\text { Perfused after } 8 \mathrm{~min} \\
\text { asphyxiation }\end{array}$} \\
\hline & & $\begin{array}{l}\text { Minimum } \\
\text { after } \\
\text { I.5-2 } \mathrm{min}\end{array}$ & $\begin{array}{c}\text { After } \\
7-10 \mathrm{~min} \\
\text { perfusion }\end{array}$ & & $\begin{array}{l}\text { After asphyx- } \\
\text { iation }\end{array}$ & $\begin{array}{l}\text { After } \\
7-\text { ro } \mathrm{min} \\
\text { perfusion }\end{array}$ \\
\hline \multirow[t]{5}{*}{$4.5 \%$} & I & $5^{8}$ & 84 & 10 & 47 & 99 \\
\hline & 2 & 61 & 92 & I I & 42 & 65 \\
\hline & 3 & 56 & 78 & 12 & 56 & $8 I$ \\
\hline & 4 & 60 & 78 & 13 & 55 & 77 \\
\hline & 5 & 51 & 79 & 14 & 49 & 106 \\
\hline Mean & & 57 & 82 & & 50 & 86 \\
\hline \multirow[t]{4}{*}{$12.5 \%$} & 6 & 69 & 76 & 15 & 40 & 107 \\
\hline & 7 & 70 & 78 & 16 & 47 & 97 \\
\hline & 8 & 57 & 92 & 17 & 44 & 93 \\
\hline & 9 & 79 & 87 & 18 & 45 & 94 \\
\hline Mean & & 69 & 83 & & 44 & 98 \\
\hline
\end{tabular}

\section{Electron microscopy}

$H A A$ perfused cortex postfixed with $\mathrm{OsO}_{4}$. Figures 2 and 3 show electron micrographs of the upper part of the molecular layer of the cerebral cortex. In agreement with Torack's ( 1965,1966$)$ observations on similar material there is considerable extracellular space in the fields of small tissue elements, probably bundles of unmyelinated fibres. However, in many places the 100-200 $\AA$ slits typical for $\mathrm{OsO}_{4}$ treated material are visible. These slits are found sometimes between the small profiles, but more often and over relatively long distances between dendritic and presynaptic structures and the glia elements. The synaptic slit has the usual width (200-250 $\AA$ ). Large, electrontransparent, presumably swollen, elements are present. They often contain mitochondria and endoplasmic reticulum which also seem enlarged. Some of the swollen structures can be identified as dendritic by synaptic contacts. Other dendritic elements identified in the same manner are relatively electron dense and do not seem swollen. Electrontransparent structures which cannot be identified by contacts with presynaptic endings may also be dendritic but the possibility cannot be excluded that some of them are glial structures. The perivascular glia usually shows a marked swelling (Fig. 4). The glial feet are electron transparent and contain sparse enlarged mitochondria and endoplasmic reticulum.

Cortex frozen $I^{\cdot} 5^{-2}$ min after the start of $H A A$ perfusion. The conductivity of the cortex passed through a minimum $1 \cdot 5^{-2}$ min after the start of HAA perfusion. In view of previous experience (Van Harreveld \& Ochs, 1956; Van Harreveld et al. 1965; Van Harreveld \& Malhotra, 1967; Van Harreveld \& Khattab, 1968) this could indicate a 
loss of extracellular material to the intracellular compartment at that time. To investigate this possibility the cortex was rapidly frozen immediately after 2 min perfusion with HAA and subjected to substitution in acetone. Figures 5 and 6 show EMs obtained in this way which are quite different from the micrographs described above. As expected there is hardly any extracellular space between the small profiles; it has been reduced to small triangles where three elements meet. Five-layered tight junctions between the plasma membranes are regularly found (Fig. 7). There are electron transparent, swollen structures in the preparation some of which can again be identified as dendritic by synaptic contacts. Other dendritic elements are not particularly electron transparent. In contrast to the observations on material fixed by HAA perfusion and $\mathrm{OsO}_{4}$ post-fixation (Fig. 4) the perivascular glia did not swell (Fig. 8). The basement membrane, however, seems unusually wide in this figure and may be slightly swollen.

The EMs of these cortices resemble strikingly those of asphyxiated cerebral cortex in which it has been shown previously that the appreciable extracellular space present in the tissue when it is frozen shortly after respiratory arrest disappears when freezing is preceded by $6-8 \mathrm{~min}$ of $\mathrm{O}_{2}$ deprivation (Van Harreveld \& Malhotra, I 967 ). These observations support the postulate that the drop in conductivity during HAA perfusion indeed indicates a loss of extracellular material to the intracellular compartment. This not only explains the paucity of extracellular material in the freeze substituted tissue but also the presence of apparently swollen structures which are believed to have taken up the extracellular fluid.

Cortex frozen after 1 5-20 min HAA perfusion. After passing through a minimum the cortical conductivity increased again during HAA perfusion. Since the drop in conductivity was accompanied by a loss of extracellular material the increase of this value could be an indication of the creation of a new extracellular space, although alternative explanations are possible. The EMs of the material frozen I 5-20 min after the start of HAA perfusion and freeze substituted in acetone support the former explanation (Figs. 9, IO). There is a great deal of space between the small elements which show rounded profiles. However, narrow slits between glial structures and dendritic and presynaptic elements are occasionally seen. The synaptic gap has the usual width. Large, apparently swollen elements are still present some of which can be identified as dendrites by the presence of synaptic contacts. Many of these elements contain enormously swollen mitochondria. Some dendritic structures show greater electron density (Fig. 9). The perivascular glia secms slightly swollen (Fig. I I). The extracellular space in these EMs resembles in its distribution that in micrographs of normal cortex frozen shortly after circulatory arrest and subjected to substitution fixation. The extracellular space is larger in the HAA-perfused material than in freeze substituted normal cortex. The swollen dendritic structures with the enormously enlarged mitochondria are typical for the tissue perfused for $15-20$ min with HAA before freezing. In normal cortex frozen shortly after circulatory arrest the electron density of all tissue elements is quite uniform (Van Harreveld et al. 1965; Van Harreveld \& Malhotra, i967).

Cortex frozen after $\mathrm{HAA}$ perfusion and post-fixation with $\mathrm{OsO}_{4}$. Figures 12-14 show 
EMs of cerebral cortex freeze substituted after $\mathrm{I}_{5}-20$ min $\mathrm{HAA}$ perfusion and $\mathrm{OsO}_{4}$ post-fixation. These EMs resemble those of cortex fixed with HAA, and post-fixed with $\mathrm{OsO}_{4}$ but not subjected subsequently to freeze substitution (Figs. 2-4). In some areas there is again considerable space between the small profiles (unmyelinated fibres) although in other regions these are separated by $100-200 \AA$ slits. Similar slits are present between the larger glial and neuronal elements. Many of the unmyelinated fibres have lost their rounded appearance and have become more or less polygonal. Swollen electron transparent structures are present some of which can be identified as dendrites; those that cannot be identified as such may be either dendritic or glial in nature. The swelling of the mitochondria is reduced as compared with those in EMs of material not post-fixed after perfusion with HAA. The perivascular glia was clearly enlarged (Fig. I4).

\section{DISCUSSION}

Perfusion with hydroxyadipaldehyde had a biphasic effect on the cortical conductivity; first it caused a drop followed after a few minutes by a rise. Large conductivity changes in tissues have in general been found to be caused by demonstrable changes in the amount of extracellular material (Van Harreveld, r966). Especially from a large and sudden drop in conductivity, as observed during the first minutes of HAA perfusion, a loss of extracellular material can be predicted with confidence. Increases of conductivity, although they can obviously be caused by an enlargement of the extracellular space, may be due to other changes. For instance, a major decrease in membrane resistance will have such an effect. The EMs of cortex frozen at the height of the initial conductivity drop and subjected to substitution fixation showed very little extracellular space and were similar to EMs of asphyxiated cerebral cortex. The appreciable extracellular space present in the normal, oxygenated cortices prepared by this method disappears during $\mathrm{O}_{2}$ deprivation (Van Harreveld et al. 1965; Van Harreveld \& Malhotra, 1967). Electron micrographs of material frozen after the subsequent rise of conductivity had taken place showed large spaces between the small tissue elements. The usually moderate conductivity increase during 7-10 min HAA perfusion (Table I) may seem insufficient to explain the very large extracellular space observed electron microscopically. As mentioned above the appearance of fluid on the cortex prevented impedance measurements for periods longer than $7-10 \mathrm{~min}$. The material used for the EMs was perfused for $\mathrm{I}^{-20} \mathrm{~min}$, however, during which an additional conductivity increase may have occurred. These considerations suggest that the conductivity changes observed are indeed related to a contraction of the extracellular space followed by the creation of new spaces.

The initial conductivity drop and loss of extracellular space during HAA perfusion is comparable with similar phenomena observed during fixation with glutaraldehyde (Nevis \& Collins, 1967; Van Harreveld \& Khattab, I968), asphyxiation of the cerebral cortex (Van Harreveld \& Ochs, 1956; Van Harreveld \& Malhotra, 1967) and during spreading depression (Leão \& Ferreira, 1953; Freygang \& Landau, 1955; Van Harreveld \& Ochs, 1957; Ranck, 1964; Van Harreveld \& Khattab, 1967). The asphyxial loss of extracellular material has been explained by the assumption that an increase in the 
sodium permeability of the plasma membrane of certain tissue elements occurs, which by physico-chemical (Donnan) forces causes a movement of extracellular $\mathrm{NaCl}$ into these cellular elements, accompanied by water to maintain osmotic equilibrium (Van Harreveld \& Ochs, I956; Van Harreveld, I 966). It seems likely that the same mechanism is responsible for the initial electrolyte and water transport into the intracellular compartment during HAA perfusion.

The subsequent rise in conductivity during HAA perfusion is of interest since it contrasts with the course of the conductivity changes observed during glutaraldehyde perfusion. The latter fixative causes a similar initial drop in conductivity which, however, is not followed by an increase during the subsequent glutaraldehyde perfusion, suggesting that the loss of extracellular space demonstrated 5 min after the start of perfusion is maintained (Van Harreveld \& Khattab, 1968). The difference in extracellular space after HAA and glutaraldehyde perfusion of $15^{-20}$ min duration may be responsible for the difference in physical properties of brain tissue treated with these fixatives; whereas glutaraldehyde fixation yields a firm tissue, treatment with HAA results in a brain which is soft and mushy. The mechanism by which fixation with these compounds results in such large differences in extracellular space can only be guessed at. One could postulate that glutaraldehyde passes both the capillary and the cell membranes with ease, whereas the penetration of HAA would be impeded by the plasma membrane. The quickly attained equal glutaraldehyde concentration in extra- and intracellular compartments would then not result in any water movement between the intra- and extracellular compartments after the initial uptake of extracellular material by the intracellular compartment has taken place. However, the presence of osmotically active HAA in the extracellular space might remove water from the intracellular compartment resulting in the creation of a new space. An additional possibility is that extensive cross linking which is a feature of glutaraldehyde fixation contributes to the firmness of the tissue perfused with this fixative and resists the formation of the new spaces.

A comparison of the EMs of cortical tissue perfused for $15^{-20}$ min with HAA and frozen either with or without post-fixation with $\mathrm{OsO}_{4}$ shows that the $\mathrm{OsO}_{4}$ treatment greatly modifies the appearance of the tissue. Many of the small tissue elements lost their rounded appearance and became polygonal, often separated by the narrow slits typical for $\mathrm{OsO}_{4}$ fixed material. The enormous enlargement of the mitochondria in the $5_{5}-20$ min HAA perfused and freeze substituted material seems to be reduced by the $\mathrm{OsO}_{4}$ post-fixation.

All the EMs whether prepared by HAA perfusion and $\mathrm{OsO}_{4}$ post-fixation or by freeze substitution during this fixation procedure show electron transparent, apparently swollen tissue elements which often can be identified as dendritic. It was postulated that these swollen structures have taken up the disappearing original extracellular material. As discussed above this transport seems to occur during the first minutes of perfusion and the swollen appearance is maintained during the subsequent perfusion and $\mathrm{OsO}_{4}$ post-fixation. The perivascular glia does not swell during the first minutes of perfusion but enlargement and electron transparency develop during the later phases of the fixation procedure. 
The suggestion posed in the introduction that the extracellular space observed in HAA perfused and $\mathrm{OsO}_{4}$ post-fixed material might be the original space in the living tissue can obviously not be maintained. Although the fluid distribution may resemble that in normal cortex frozen shortly after circulatory arrest, in the $\mathrm{HAA}$ and $\mathrm{OsO}_{4}$ treated material this state is reached through a phase of extreme paucity of extracellular material. The dissection of the fixation process by freeze substitution demonstrated a sequence of major changes of the fluid distribution which precludes any direct relationship between the spaces in the normal and the fixed tissues.

This investigation was supported by a grant from the National Science Foundation (GB 6698).

\section{REFERENCES}

Freeman, J. A. \& Spurlock, B. O. (1962) A new epoxy embedment for electron microscopy. 7. Cell Biol. 13, 437-443.

FreYGang, Jr., W. H. \& Landau, W. M. (1955). Some relations between resistivity and electrical activity in the cerebral cortex of the cat. F. cell. comp. Physiol. 45, 377-392.

Leño, A. A. P. \& Ferreira, H. M. (1953). Altração da impedancia elétrica no decurso da depressão alastrante de atividade do córtex cerebral. Anais Acad. bras. Cienc. 25, 259-266.

Nevis, A. H. \& Collins, G. H. (1967). Electrical impedance and volume changes in brain during preparation for electron microscopy. Brain Res. 5, 57-85.

RANCK, J. B. (1964). Specific impedance of cerebral cortex during spreading depression, and an analysis of neuronal, neuroglial, and interstitial contributions. Expl Neurol. 9, 1-16.

REYNoLDS, E. S. (1963). The use of lead citrate at high $\mathrm{pH}$ as an electron-opaque stain in electron microscopy. 7. Cell Biol. 17, 208-212.

Richardson, K. S., JARETT, L. \& FinKE, E. H. (1960). Embedding in epoxy resins for ultrathin sectioning in electron microscopy. Stain Technol. 35, 313-323.

Torack, R. M. (1965). The extracellular space of rat brain following perfusion fixation with glutaraldehyde and hydroxyadipaldehyde. $Z$. Zellforsch. mikrosk. Anat. 66, 352-364.

TORACK, R. M. (1966). The penetration of thorotrast into brain spaces following osmium, glutaraldehyde, and hydroxyadipaldehyde fixation. F. Ultrastruct. Res. 14, 590-601.

VAN HARREVELD, A. (1957). Changes in volume of cortical neuronal elements during asphyxiation. Am. F. Physiol. 191, 233-242.

VAN HARREVELD, A. (1958). Changes in diameter of apical dendrites during spreading depression. Am. F. Physiol. 192, 457-463.

VAN HaRREveld, A. (1961). Asphyxial changes in the cerebellar cortex. F. cell. comp. Physiol. 57, IOI-110.

VAN HaRReveld, A. (1966). Brain Tissue Electrolytes. New York and London: Butterworth.

Van Harreveld, A. \& Crowell, J. (1964). Electron microscopy after rapid freezing on a metal surface and substitution fixation. Anat. Rec. 149, 381-386.

Van Harreveld, A., Crowell, J. \& Malhotra, S. K. (1965). A study of extracellular space in central nervous tissue by freeze-substitution. F. Cell Biol. 25, I 1 7 -1 37 .

Van HaRreveld, A. \& KhatTaB, F. I. (I967). Changes in cortical extracellular space during spreading depression, investigated with the electron microscope. $\mathcal{F}$. Neurophysiol. 30, 91 I -929 .

Van Harreveld, A. \& Khattab, F. I. (1968). Perfusion fixation with glutaraldehyde and postfixation with osmium tetroxide for electron microscopy. F. Cell Sci. 3, 579-594.

Van Harreveld, A. \& Malhotra, S. K. (1967). Extracellular space in the cerebral cortex of the mouse. F. Anat. 10r, 197-207.

Van HaRReveld, A. \& OCHS, S. (1956). Cerebral impedance changes after circulatory arrest. Am. F. Physiol. 187, 180-192.

Van HARREVELD, A. \& OCHS, S. (1957). Electrical and vascular concomitants of spreading depression. Am. J. Physiol. 189, I 59-166.

VAN HaRReveld, A. \& SCHADÉ, J. P. (1959). Chloride movements in cerebral cortex after circulatory arrest and during spreading depression. . cell. comp. Physiol. 54, 65-84.

(Received 29 May 1968) 


\section{ABBREVIATIONS ON PLATES}

$\begin{array}{ll}b m & \text { basement membrane } \\ d & \text { dendrite } \\ e & \text { endothelium } \\ e r & \text { endoplasmic reticulum }\end{array}$

$g \quad$ glia

$m$ mitochondrion

pr presynaptic terminal

Figs. 2-4. Surface of the molecular layer of the cerebral cortex perfused with hydroxyadipaldehyde and post-fixed with osmium tetroxide. Swollen dendrites, identified by synaptic contacts, with moderately enlarged mitochondria and endoplasmic reticulum are present. Some dendrites do not appear swollen. Swollen structures which cannot be identified by synaptic contacts may be either dendritic or glial processes. Narrow slits are found between glia processes and the dendritic and presynaptic structures. Between the small tissue elements which have in general a polygonal shape and probably are unmyelinated axons there are at many places similar narrow slits but also larger areas of extracellular space (arrows). Fig. 4 shows a capillary surrounded by swollen perivascular glia. Calibration line of all three figures is $\mathrm{I} \mu$. 


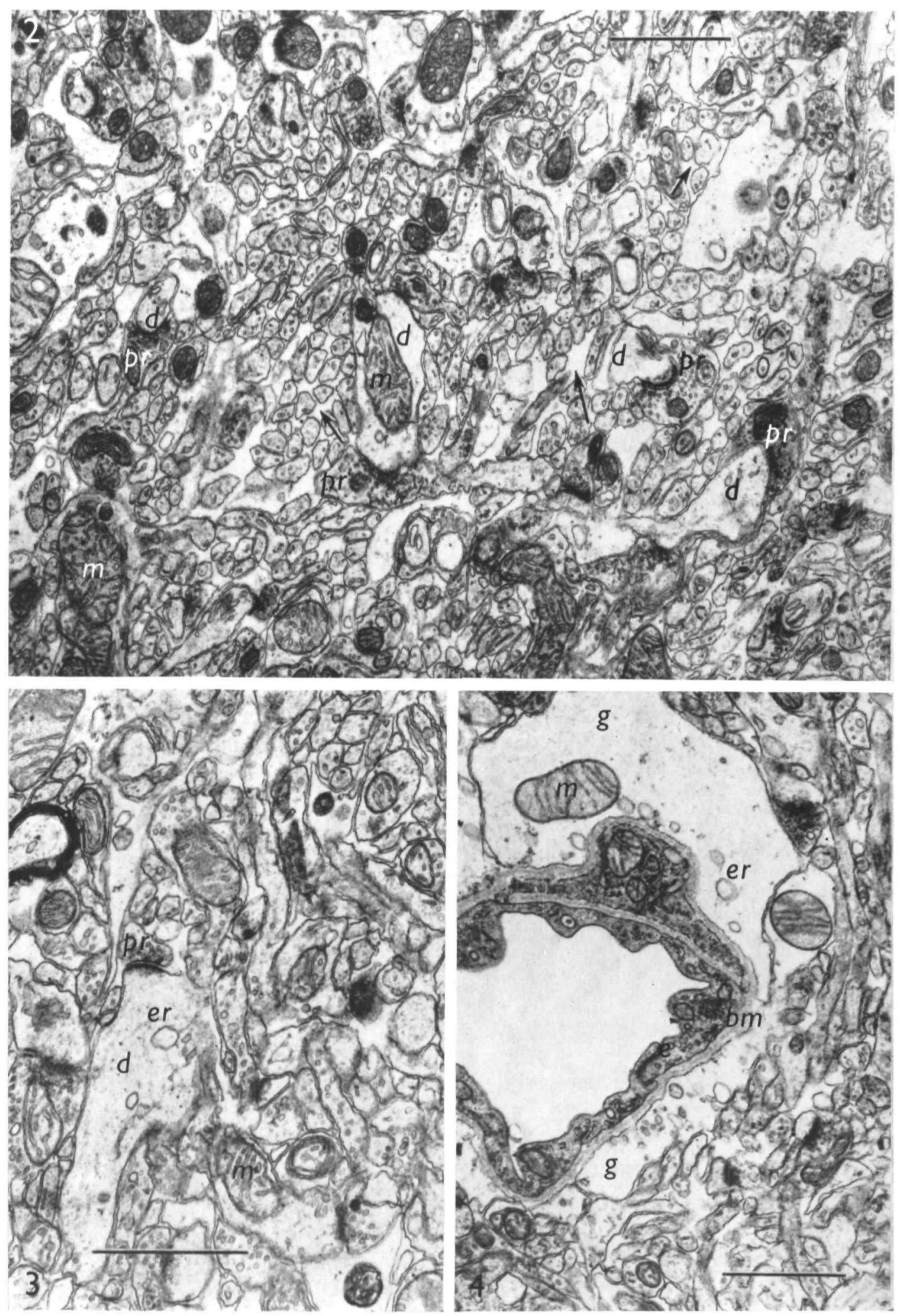


Figs. 5-8. Surface of the cerebral cortical molecular layer frozen after 2 min of HAA perfusion and subjected to substitution fixation. Part of the surface glia is shown in Fig. 5. Figures 5 and 6 show electron transparent, swollen dendritic structures identified by synaptic contacts, others show a rather high electron density. The tissue is characterized by a paucity of extracellular material, which is restricted to small, often triangular areas where three tissue elements meet. The plasma membranes are so closely approximated that 5-layered tight junctions are formed (arrows in Fig. 7). Part of a blood vessel is shown in Fig. 8. The perivascular glia has a high electron density and is not swollen. The basement membrane seems unusually wide and may be slightly swollen. Calibration lines in Figs. 5, 6 and 8 indicate $\mathrm{I} \mu$; in Fig. 7 the line is $0 \cdot 2 \mu$. 

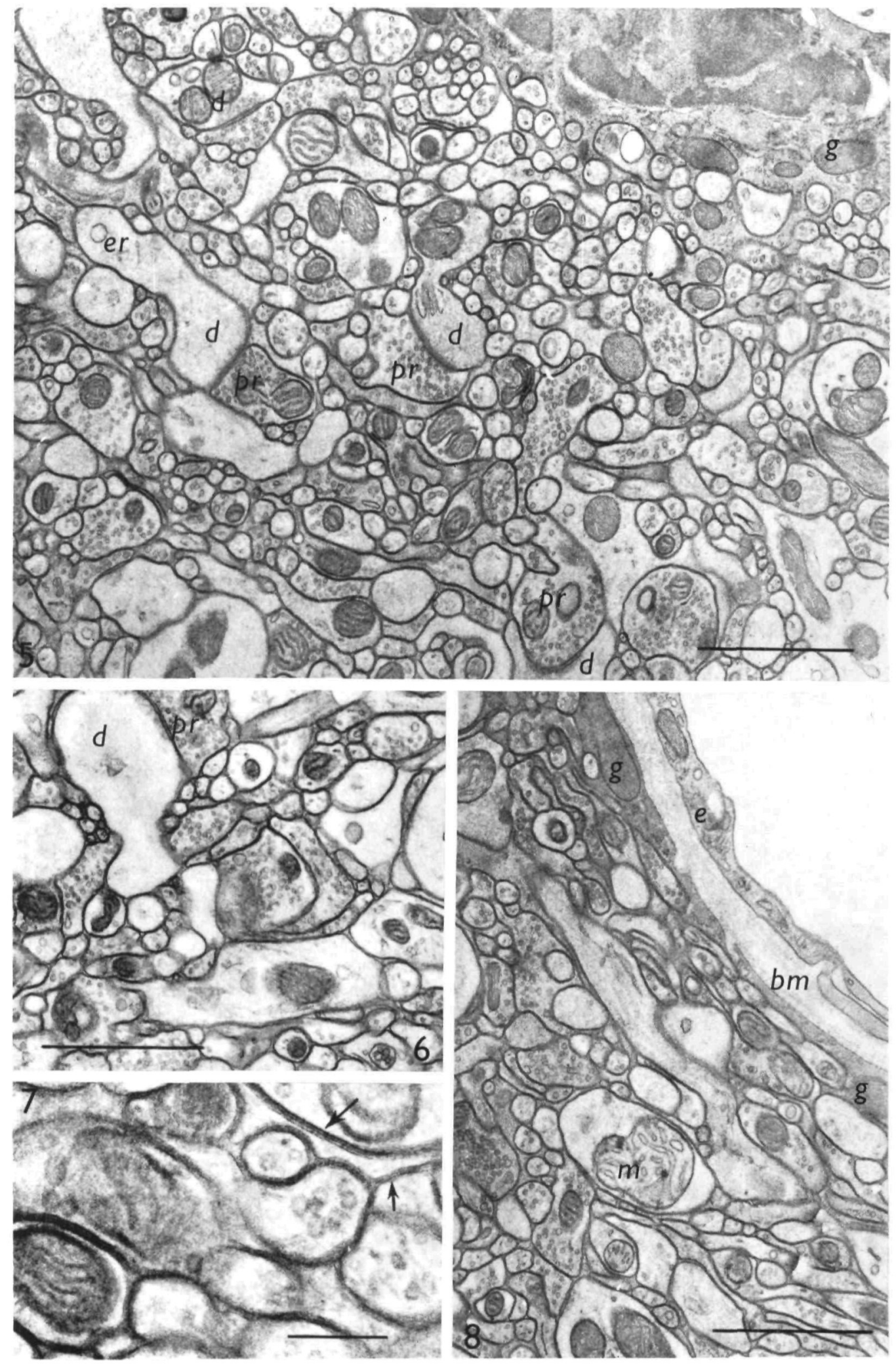
Figs. 9-I I. Surface of the cerebral cortex frozen after perfusion with glutaraldehyde for $20 \mathrm{~min}$ and subjected to freeze substitution. Enlarged structures are present which by their synaptic contacts can be identified as dendritic. Many of these contain enormously swollen mitochondria. An abundant extracellular space (arrows) is present between the unmyelinated axons which have a rounded profile. Fig. I I shows a capillary. Its perivascular glia may be somewhat swollen. The calibration lines in all three figures indicate $\mathrm{I} \mu$. 


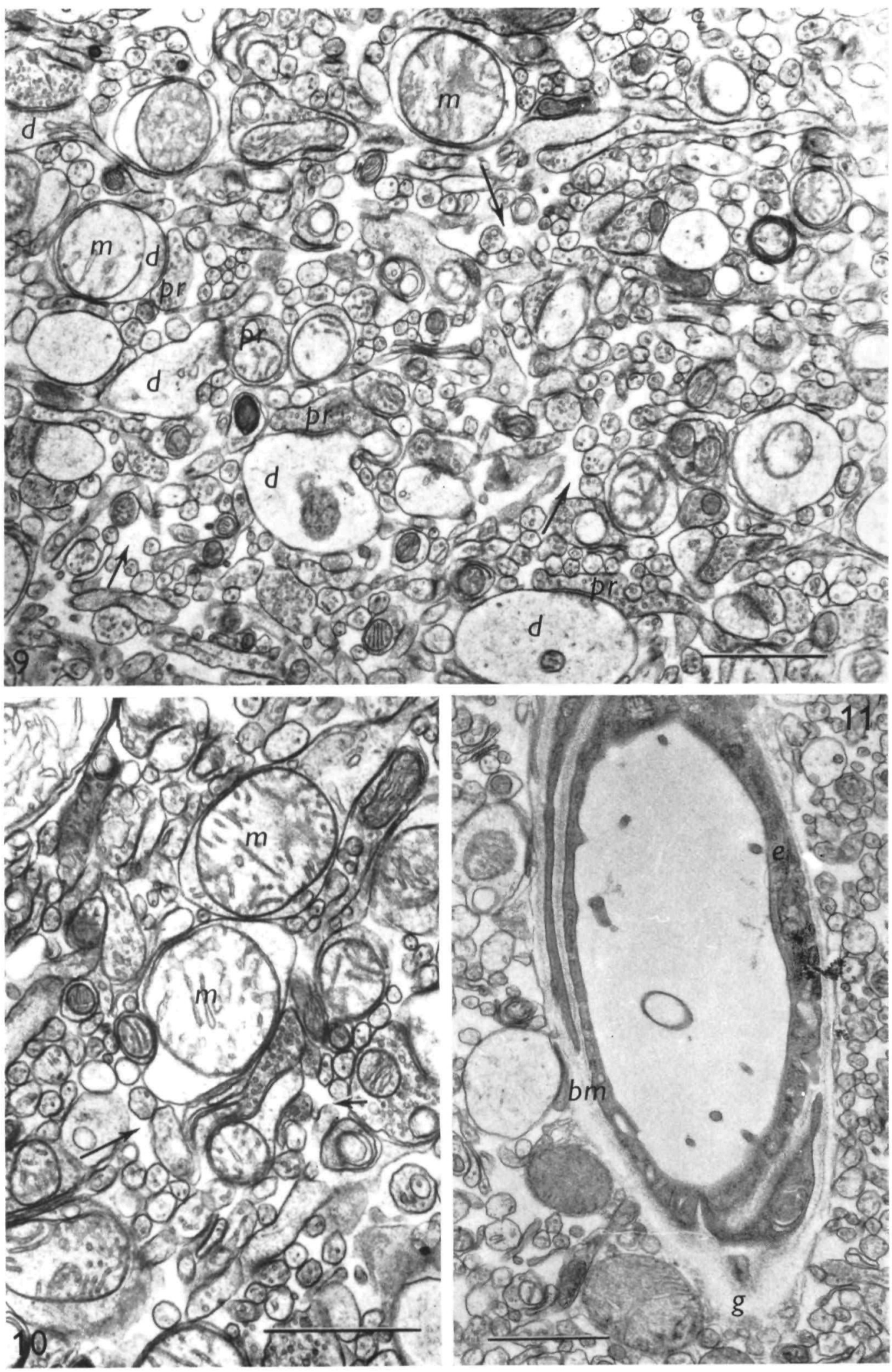


Figs. I 2-I4. Surface of the cortical molecular layer frozen after HAA perfusion for $20 \mathrm{~min}$ and post-fixation with $\mathrm{OsO}_{4}$. Some of the dendritic structures identified by synaptic contacts are moderately swollen. Narrow extracellular slits are found between most tissue elements, although fields of unmyelinated fibres often show larger extracellular spaces (arrows). Figure i 4 shows a capillary with swollen perivascular glia. These micrographs resemble in their general appearance those shown in Figs. 2-4 which were fixed in the same way but not subjected to freeze substitution. Calibration in all figures is $\mathrm{I} \mu$. 


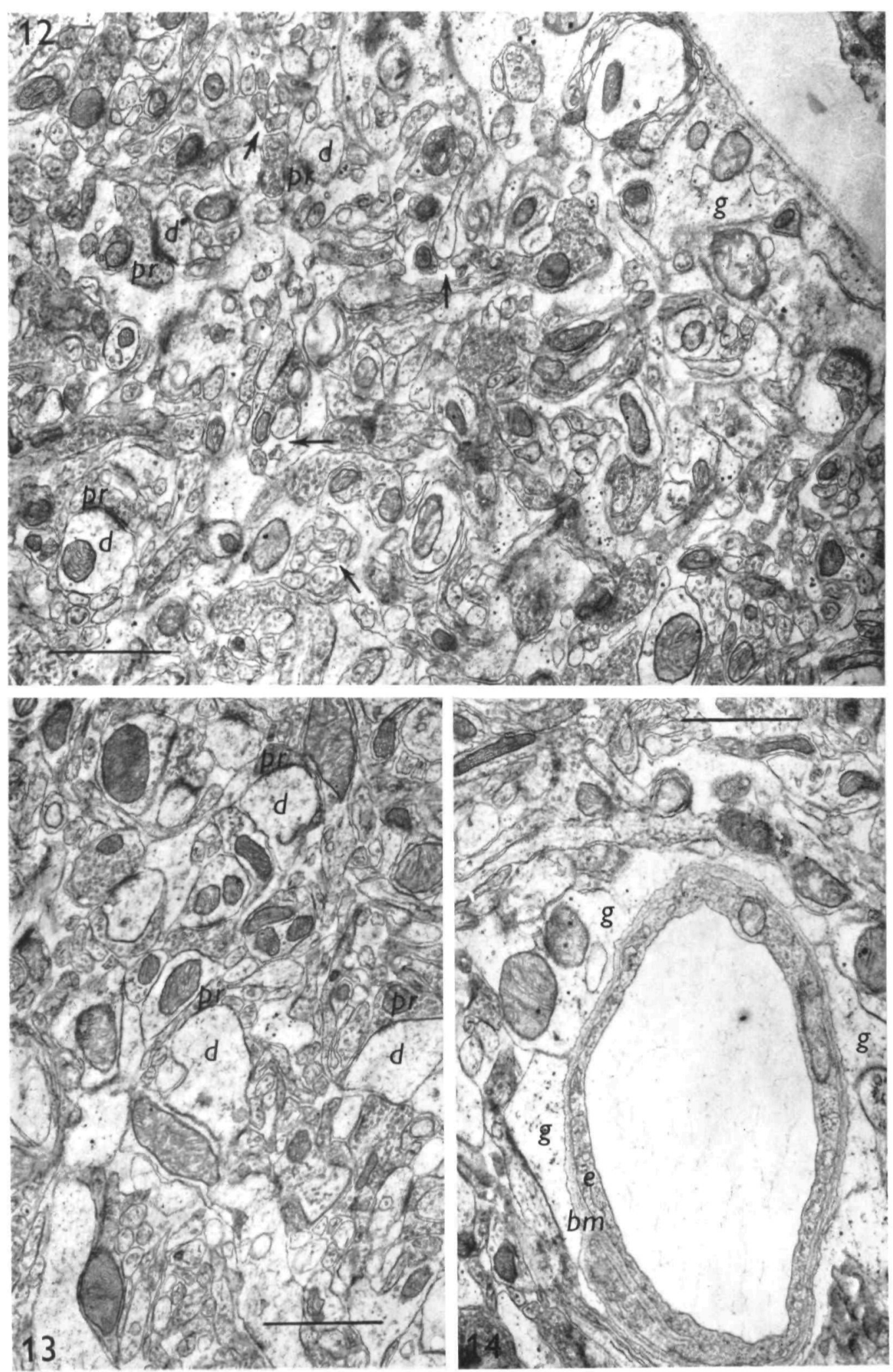


